$\underline{\text { Review Article }}$

\title{
DIFFERENT TYPES OF NANOCARRIERS FOR TARGETED TREATMENT OF HIV/AIDS
}

\author{
ANURUPA OJAH ${ }^{*}$, BIPULNATH ${ }^{2}$, TRISHNA DAS ${ }^{3}$
}

1,2Department of Pharmaceutics, Girijananda Chowdhury Institute of Pharmaceutical Science, Hatkhowapara, Azara, Guwahati-17, ${ }^{3}$ Department of Pharmacognosy, Girijananda Chowdhury Institute of Pharmaceutical Science, Azara, Hatkhowapara, Guwahati-17 Email: anurupaoza78@gmail.com

Received: 17 Apr 2019, Revised and Accepted: 26 Jun 2019

\begin{abstract}
AIDS is a major challenge in the 21st century. AIDS, stands for Acquired Immune Deficiency Syndrome, is a serious health problem and a fastspreading incurable disease. It is a disorder of the cell-mediated immune system of the body. It is localized in certain inaccessible compartment of the body such as the CNS, lymphatic system and in the macrophages, where it cannot be reached by the majority of the therapeutic agent in adequate concentration or in which the therapeutic agents cannot for the necessary duration. HIV/AIDS infection are reducing incidence with the advent of Highly Active Antiretroviral Therapy (HAART). There remain a number of challenges including drug resistance, anatomical barriers to antiretroviral therapy. To overcome these different nanocarriers with the stimuli-responsive release are proposed for antiretroviral agents. Nanocarrier and those optimized for cancer chemotherapy. These include the development nanoparticles capable of passive and active targeting as well as those that are responsive to various internal and externl triggers. Nanocarriers can also provide many of the advantages of lipid system. Liposome, solid lipid nanoparticle, nanoemulsion, dendrimers, polymeric nanoparticle are the example of drug delivery systems that are under investigation for the treatment of HIV/AIDS. This review aims to summarize the ability of nanocarrier technology to provide a rational approach for anti-HIV therapy.
\end{abstract}

Keywords: AIDS, HIV, Nanocarrier technology, Targeted drug delivery

(C) 2019 The Authors. Published by Innovare Academic Sciences Pvt Ltd. This is an open access article under the CC BY license (http://creativecommons.org/licenses/by/4.0/] DOI: http://dx.doi.org/10.22159/ijcpr.2019v11i4.34956

\section{INTRODUCTION}

Nanocarrier is nanomaterial being used as a transport module for another substance, such as a drug. Nano-based drug delivery systems, where the formulation is restricted to $<1000 \mathrm{~nm}$ in diameter (typically 100-500 nm), can be fabricated to protectdrug molecules and to preferentially target the drugs to specific anatomical and cellular targets [1]. In addition, nanocarrier-based systems also offer a unique feature in terms of drug targeting, which makes this system the most versatile option, especially for HIV/AIDS. AIDS, stands for Acquired Immune Deficiency Syndrome, is a serious health problem and a fastspreading incurable disease. It was first noticed in USA in 1981. It is a disorder of the cell-mediated immune system of the body. There is a reduction in the number of helper T-cells which stimulate antibody production by B-cell. AIDS is caused by Human immunodeficiency virus (HIV) which is an RNA virus. Virus was named variously as human cell leukemia virus-III (HCLV-III), human T-lymphotrophic virus III (HTLV-III), lymphadenopathy-associated virus (LAV), and ultimately HIV in 1986 by International Committee on taxonomy of virus. HIV is having two species, namely, HIV-1 and HIV-2. HIV-1 is responsible for common infection around the globe but HIV-2 is more prominent in West Africa. HIV-2takes longer time to develop immunodeficiency in patients compared to HIV-1. HIV is having a special protein namely "Viral gp 120 protein" that binds to transmembrane receptor CD4+or one of the chemokine receptor CCR5 or CXCR4 of the host cell and infects the cell [2].

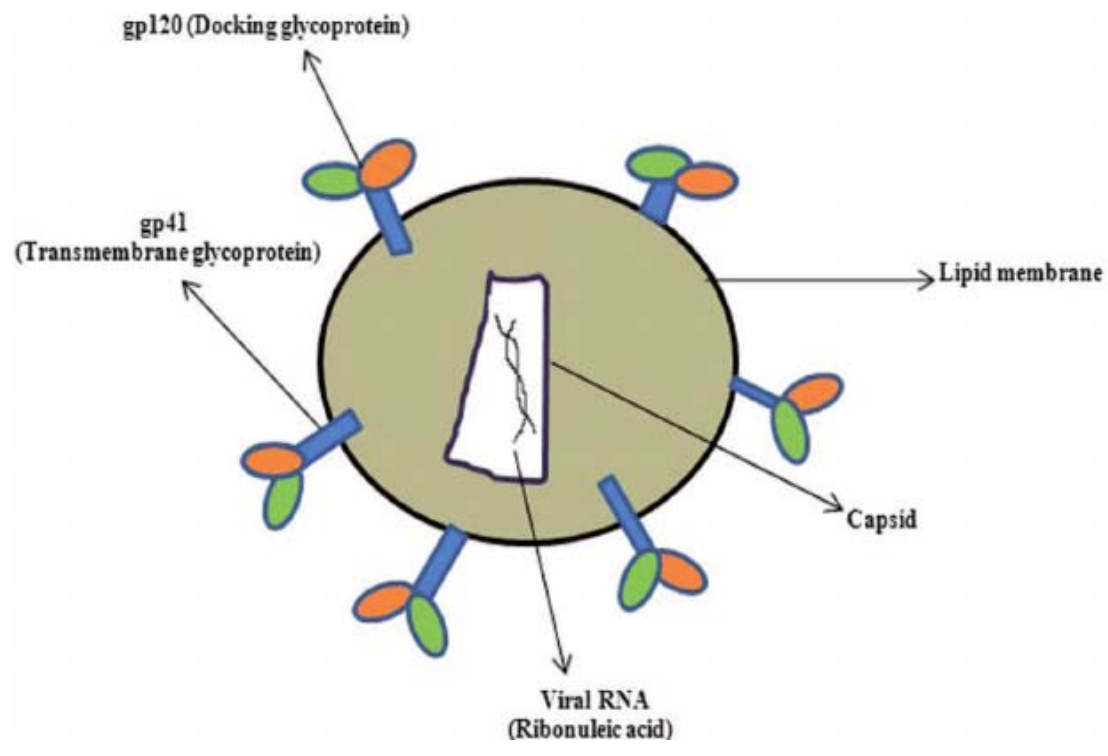

Fig. 1: Structural arrangement of HIV 


\section{Nanocarrier technology for HIV/AIDS therapy}

For HIV/AIDS therapeutics, nanocarriers have been reported to be used for transporting the drugs to reservoir compartments in the body, in particular to the macrophages, lymphatic system and/or CNS. Lipid and polymeric nanosystems are typically used for delivery to overcome cellular and anatomical barriers. Lipid nanosystems usually comprise a bilayer or multilayer system wherein one of the layers contains lipids (outer layer), whereas the core layer encapsulates the drug substance (inner layer). The lipid nanosystems not only protect the drug but also provide flexibility to encapsulate most hydrophobic pharmaceutical compounds. Enhanced lipophilicity and surface engineering of lipid nanocarriers can significantly influence the transport of the drugs across the mucosa and the physiological and anatomical barriers that are present in the body. These delivery systems can be designed in a tailor-made format, depending on the need to provide typical surface characteristics for targeting drug compounds. Polymeric nanocarriers can also provide many of the advantages of lipid systems and can be modified for target specificity. Liposomes, solid- lipid nanoparticles (SLNs), nanoemulsion, dendrimers and polymeric nanocarriers are some of the examples of widely used nanoparticulate drug delivery systems [3].

\section{Liposomes}

Liposomes are microscopic vesicles consisting of one or more phospholipid bilayers which usually surround anaqueous core. Liposomes are having size between $25 \mathrm{~nm}$ and several microns. In their preparation, natural or synthetic phospholipids along with cholesterol are used. Additionally, they may have other lipids or proteins. Hydrophilic drugs are entrapped in the aqueous core while hydrophobic drugs are incorporated in the lipid bilayers. When liposomes enter in the human body, they are recognized as the foreign bodies and easily taken up by the mononuclear phagocytic cells. HIV is present in mononuclear phagocytic cells of an infected patient, so liposomes represent suitable carriers for targeting anti-HIV drugs to the infected cells. Thus, liposomes can improve the efficacy of of antiHIV drugs and reduce their side effects. Selective uptake behavior of liposomes by mononuclear phagocytes is given in fig. 2 .

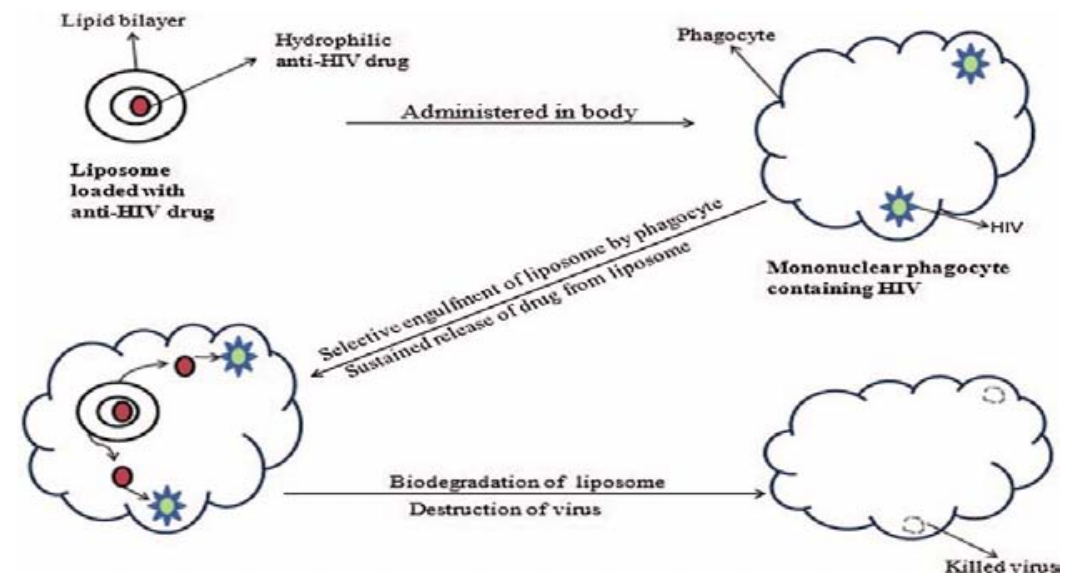

Fig. 2: Selective uptake of drug-loaded liposomes by mononuclear phagocytes

\section{Solid lipid nanoparticle}

SLNs have been widely employed for delivering drugs to the CNS by circumventing the blood-brain barrier. SLNs are 200-500 nm in diameter and can be prepared from solid or semi-solid fatty acids (e. g., cetyl palmitate, salts of myristic acid), and stabilisation of dispersions with emulsifiers and co-emulsifiers, such as polysorbates, poloxamers, fatty acidco-esters, lecithin and bile salts. SLNs are prepared by heating the components to cause them to liquefy and undergo emulsification in an aqueous media. On cooling, the solid nanocarriersseparate out and can be easily filtered and dried [4]. Themajority of drugs can be incorporated into the SLNs in the theliquid system during the preparation. SLNs can also be modified for size and surface charges in order to achieve site-specific drug delivery designed for immediate or prolonged release. Finally, SLN can be engineered to release their payload in response to a specific external trigger, such as temperature or pH. Solid lipid nanoparticle is given in fig. 3 .

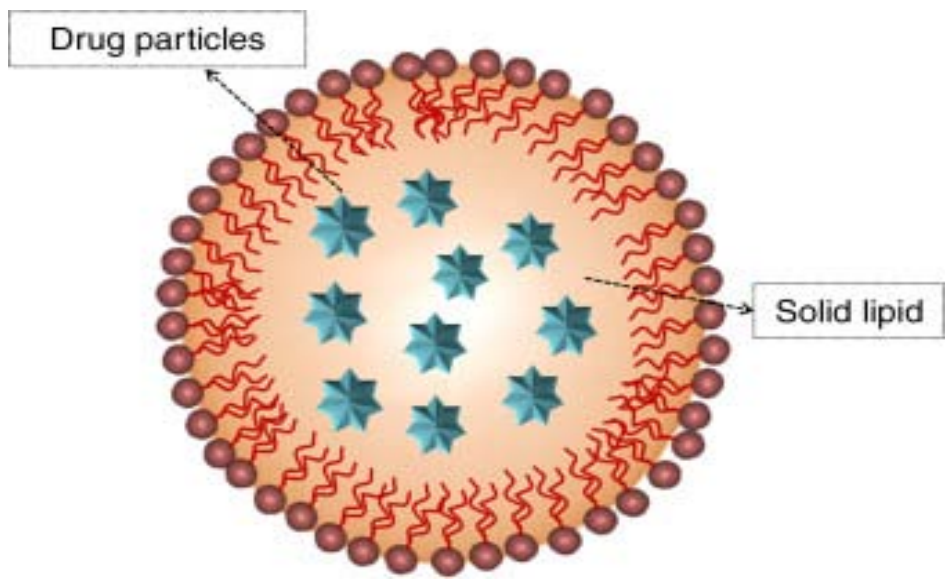

Fig. 3: Solid lipid nanoparticle 


\section{Nanoemulsion}

Nanoemulsions are heterogeneous liquid dispersions where the the droplet size of the internal phase is reduced to a nanometer scale. Nanoemulsions exist in a broad variety of forms that are designed using different types of oils and a variety of emulsifiers to render them specific for the given application. The oil-droplets of nanoemulsions are generally considered to be in the thesize range of $\leq 400 \mathrm{~nm}$ in diameter. The emulsions can exist as water-in-oil and oil-in-water forms, wherein the core of the particle is usually either water or oil. The long-term properties of the internal phase are dependent on the composition of the the adsorbed surface-active agent (surfactant) lying at the dispersed droplet interface with the dispersion medium. This has an impact on the partitioning and extraction of droplet contents. Nanoemulsions are thermodynamically stable particles and can be characterized by having very low surface tension [5]. The small size of oil droplets leads to a very large surface-area-to-volume ratio for delivery and to better physical stability of the formulation. The choice of oils with specific fatty acid composition can influence transport properties, as well as a therapeutic activity. The surface properties and chemistry can strongly influence in vivo behavior of nanoemulsions. Further developments in nanoemulsion-engineering technologies are likely to lead to much greater use as carriers for targeted drug delivery for different disease conditions, as well asfor newer generations of therapeutic agents.

\section{Dendrimers}

A dendrimer is a polymeric nanostructure $(<100 \mathrm{~nm})$ built around a core unit. There are several branching units around the core units in a layer-by-layer fashion which defines the growth, size, and the microenvironment within the dendrime. Dendrimers are composed of a large number of smaller units known as dendrons. Dendrons are formed after removal of core units and can be divided into (empty) core, the interior (branching units), and the periphery (end groups).

The empty space lying inside the dendrons can be used for the entrapment of drug molecules for solubilization, controlled release, targeting, or protection from surrounding degrading environment. Dendrimers show various advantages like uniform particle size, ability to bind a variety of targeting agents to their high-density peripheral functional groups, and poly-valency of the end groups which helps in binding to diverse receptors [6]. Fig. 4 shows that how dendrimers can be effective nontargeted delivery of anti-HIV drugs.

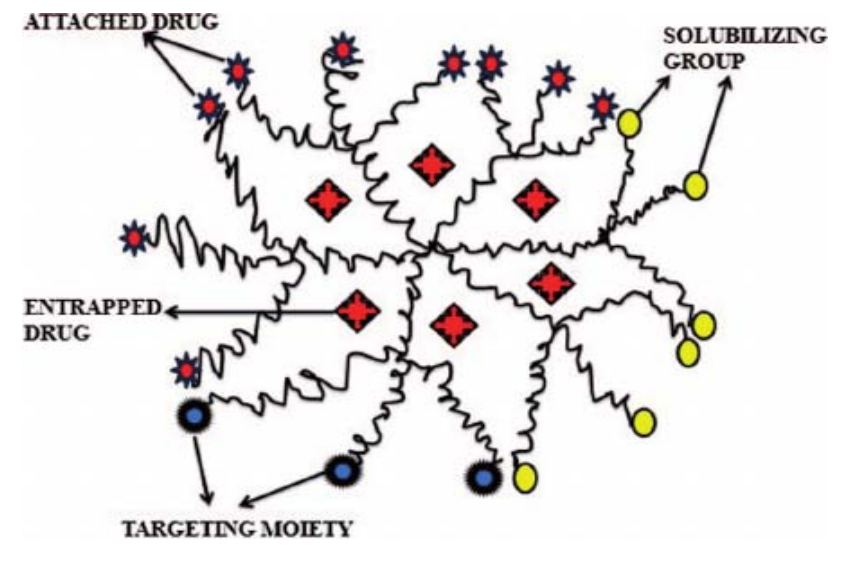

Fig. 4: Polymeric dendrimers in anti-HIV drug targeting

\section{Polymeric nanoparticle}

Due to their versatility, polymers offer a unique opportunity for the development of nanocarrier systems. Nanoparticles are defined as solid, colloidal particles consisting of eithernon-degradable or biodegradable polymeric components. Nanoparticles have a particle size typically ranging from 200-800 $\mathrm{nm}$. A large variety of synthetic and natural polymeric materials, such as poly(D,L-lactide-co-glycolide),poly( $\varepsilon$ caprolactone), chitosan, albumin and gelatin are used for the preparation of nanoparticles. The drug is either encapsulated or adsorbed on the nanoparticle surface. As with liposomes, polymeric nanoparticles also can be tailor-made with regard to the surface charge and particle size, based on the need. The particles can be coated by using surfactants for immediate-release preparations. However, the the potential problem with nanoparticles is aggregation that canbe decreased by surface modification with amphipathic copolymers, such as poloxamines or poloxamers [7]. Surface modification with PEG or poly (ethylene oxide), using a variety of poly(ethylene oxide)-containing copolymers, can prolongthe circulation times in the plasma and allow for passive delivery [8]. The efficiency of particle uptake by peripheral blood mononuclear cells can be altered by varying the concentration of block polymers on the nanoparticle surface to regulate phagocytosis and preferential uptake by the reticuloendothelial system [9]. Reports in the literature cite that polymeric nanoparticles can be used for a wide spectrum of drug delivery applications, including inside macrophages andin the CNS $[10,11]$. Nanoparticle-based carrier systems can also be used to alter the drug release pattern and, in turn, to alter the biodistribution and pharmacokinetics of the drug. Polymeric nanoparticle is given in fig. 5 .

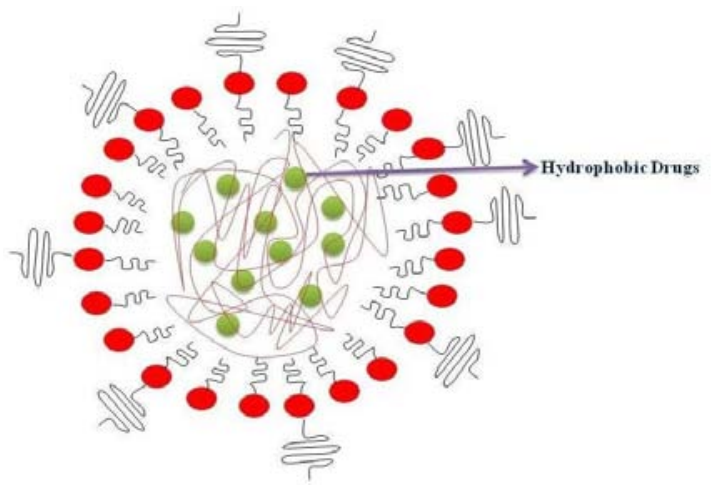

Fig. 5: Polymeric nanoparticle 


\section{CONCLUSION}

In this article, we have discussed the ability of nanocarrier technology to provide a rational approach for anti-HIV therapy. HIV/AIDS therapy represents a significant challenge at different anatomical and cellular levels. Nanotechnology offers tremendous potential for the appropriate management of HIV/AIDS. In addition to increasing the ability to target therapies, the nanocarrier formulations can be designed to reduce the effective dose, combine more than one agent in a single formulation, and provide rationale delivery mechanisms for attacking the virus at reservoir sites. Nanocarrier-based drug delivery systems, such as liposomes, solid lipid nanoparticle, nanoemulsions, dendrimers and polymeric nanotechnology-based products, have already been tried in various preclinical studies and have been shown to improve delivery efficacy to the reservoirs. The delivery of the drug at targeted sites and the maintenance of therapeutic drug concentrations for prolonged periods can help in an appreciable reduction of the viral load.

\section{AUTHORS CONTRIBUTIONS}

All the author have contributed equally

\section{CONFLICT OF INTERESTS}

\section{Declare none}

\section{REFERENCES}

1. Vyas TK, Shah L, Mansoor MA. Nanoparticulate drug carriers for delivery of HIV/AIDS therapy to viral reservoir sites. Expert Opinion 2006;3:613-8.
2. Kumar L, Verma S, Prasad DP. Nanotechnology: a magic bullet for HIV AIDS treatment. Artificial Cells Nanomed Biotechnol 2015;43:71-86.

3. Parboosing R, Maguire GEM, Govender P. Nanotechnology and the treatment of HIV infection. Viruses 2012;11:488-520.

4. Bummer PM. Physical-chemical considerations of lipid-based oral drug delivery-solid lipid nanoparticles. Crit Rev Ther Drug Carrier Syst 2004;21:1-20.

5. Wynn HE, Brundage RC, Fletcher CV. Clinical implications of CNS penetration of antiretroviral drugs. CNS Drugs 2002;16:595-609.

6. Villalonga BC, Micha SM, Steele BR, Georgopolous A Dendrimers as biopharmaceuticals: synthesis and properties. Curr Top Med Chem 2008;8:1294-309.

7. Shenoy D, Little S, Langer R. Poly (ethylene oxide)-modified poly ( $\beta$-amino ester) nanoparticles as a $\mathrm{pH}$-sensitive system for tumor-targeted delivery of hydrophobic drugs: part 2. M In vivo distribution and tumor localization studies. Pharm Res 2005;22:2107-14.

8. Torchilin VP. Lipid-core micelles for targeted drug delivery. Curr Drug Delivery 2005;2:319-27.

9. Roney C, Kulkarni P, Arora V. Targeted nanoparticles for drug delivery through the blood-brain barrier for Alzheimer's disease. J Controlled Release 2005;108:193-214.

10. Basu MK, LALA S. Macrophage-specific drug delivery in experimental leishmaniasis. Curr Mol Med 2004;4:681-9.

11. Adhikary RR, Morea P, Banerjeea R. Smart nanoparticles as targeting platforms for HIV infections. Royal Soc Chem 2004;3:1-16. 